\title{
C-type lectin receptors and RIG-I-like receptors: new points on the oncogenomics map
}

This article was published in the following Dove Press journal:

Cancer Management and Research

2I February 2012

Number of times this article has been viewed

\author{
Anton G Kutikhin \\ Arseniy E Yuzhalin \\ Department of Epidemiology, \\ Kemerovo State Medical Academy, \\ Kemerovo, Russian Federation
}

Correspondence: Anton G Kutikhin Darvina Street 2-9, Kemerovo, Russian Federation, 650025

Tel $+7384275 \quad 1744$

Fax $+7384275 \quad 1744$

Email antonkutikhin@gmail.com
Abstract: The group of pattern recognition receptors includes families of Toll-like receptors, NOD-like receptors, C-type lectin receptors, and RIG-I-like receptors. They are key sensors for a number of infectious agents, some of which are oncogenic, and they launch an immune response against them, normally promoting their eradication. Inherited variations in genes encoding these receptors and proteins and their signaling pathways may affect their function, possibly modulating cancer risk and features of cancer progression. There are numerous studies investigating the association of single nucleotide polymorphisms within or near genes encoding Toll-like receptors and NOD-like receptors, cancer risk, and features of cancer progression. However, there is an almost total absence of articles analyzing the correlation between polymorphisms of genes encoding C-type lectin receptors and RIG-I-like receptors and cancer risk or progression. Nevertheless, there is some evidence supporting the hypothesis that inherited C-type lectin receptor and RIG-I-like receptor variants can be associated with increased cancer risk. Certain C-type lectin receptors and RIG-I-like receptors recognize pathogen-associated molecular patterns of potentially oncogenic infectious agents, and certain polymorphisms of genes encoding C-type lectin receptors and RIG-I-like receptors may have functional consequences at the molecular level that can lead to association of such single nucleotide polymorphisms with risk or progression of some diseases that may modulate cancer risk, so these gene polymorphisms may affect cancer risk indirectly. Polymorphisms of genes encoding C-type lectin receptors and RIG-I-like receptors thereby may be correlated with a risk of lung, oral, esophageal, gastric, colorectal, and liver cancer, as well as nasopharyngeal carcinoma, glioblastoma, multiple myeloma, and lymphoma. The list of the most promising polymorphisms for oncogenomic investigations may include rs1926736, rs2478577, rs2437257, rs691005, rs2287886, rs735239, rs4804803, rs16910526, rs36055726, rs11795404, and rs10813831.

Keywords: C-type lectin receptors, RIG-I-like receptors, cancer, single nucleotide polymorphisms, genetic variation, inflammation

\section{Brief description of pattern recognition receptors}

Pattern recognition receptors directly recognize common antigen determinants of virtually all classes of pathogens (so-called pathogen-associated molecular patterns, or PAMPs). ${ }^{1-4}$ In addition, they recognize endogenous ligands, usually releasing during cell stress and known as damage-associated molecular patterns. ${ }^{1-4}$ As a result of ligand recognition, pattern recognition receptors initiate an immune response via specific intracellular signaling pathways, and so have a key role in initiation and promotion of septic and aseptic inflammation. ${ }^{1-4}$ Pattern recognition receptors also have a number of other vital functions apart from participation in the immune response, in that they may regulate many aspects of cell proliferation, survival, apoptosis, autophagy, generation 
of reactive oxygen species, pyroptosis, angiogenesis, and, consequently, tissue remodeling and repair. ${ }^{1-4}$ There are four main groups of pattern recognition receptors, ie, Toll-like receptors, NOD-like receptors, C-type lectin receptors, and RIG-I-like receptors, and genes encoding them are broadly expressed, eg, in epithelial cells, endothelial cells, keratinocytes, lymphocytes, granulocytes, fibroblasts, and neurons. ${ }^{1-4}$ A summary of the most modern conceptual data about members of these groups and about their structure and function can be obtained from recent comprehensive reviews by Kawai and Akira, ${ }^{1}$ Elinav et al, ${ }^{2}$ Osorio et al, ${ }^{3}$ and Loo and Gale. ${ }^{4}$

The completion of the human genome project and widespread distribution of genotyping technologies have led to an enormous number of studies devoted to associating inherited gene polymorphisms with various diseases. Single nucleotide polymorphisms may result in amino acid substitutions altering protein function or splicing, and they can also change the structure of enhancer sequences during splicing ${ }^{5}$ and affect mRNA stability. ${ }^{6}$ Single nucleotide polymorphisms may alter transcription factor binding motifs, change the efficacy of enhancer or repressor elements, ${ }^{7}$ and alter the structure of translation initiation codons that may lead to downregulation of wild-type transcripts. ${ }^{8}$ Gene polymorphisms located in leucine-rich repeats constituting ectodomains of many pattern recognition receptors may affect the ability of these receptors to bind pathogens they normally recognize, ${ }^{9}$ single nucleotide polymorphisms in transmembrane domains can lead to defects of intracellular receptor transport that prevent receptors localizing to the cell membrane, ${ }^{10}$ and, finally, polymorphisms in the cytosolic domains may result in altered interactions with adaptor proteins or in disrupted receptor dimerization. Therefore, there are many avenues by which single nucleotide polymorphisms may alter pattern recognition receptor expression and activity. Because pattern recognition receptors recognize a number of oncogenic infectious agents and launch an immune response against them, inherited variation in their structure may modulate cancer risk and, possibly, influence cancer progression. In addition, pattern recognition receptors bind a lot of endogenous ligands, ${ }^{1-4}$ so polymorphisms of genes encoding them can affect risk and/or progression of some autoimmune disorders and, consequently, cancer risk and/or progression, given that there is a fundamental and epidemiological association between many autoimmune diseases and cancer risk.

\section{The problem}

Although there are a lot of studies investigating the association between single nucleotide polymorphisms in genes encoding Toll-like receptors and NOD-like receptors and the risk and features of cancer progression, there is an almost complete absence of articles analyzing the correlation between polymorphisms of genes encoding C-type lectin receptors and RIG-I-like receptors and cancer risk or progression. This can be explained by the fact that the first wave of studies devoted to the association of polymorphisms of genes encoding Toll-like receptors and NOD-like receptors with cancer risk appeared only in 2004, and the number of such papers was relatively small until 2008. In addition, known hypotheses about the infectious agents causing human cancer and their recognition by pattern recognition receptors suggested that Toll-like receptors and NOD-like receptors should play a major role in the immune response against biological carcinogens. However, more recent findings concerning specific potentially carcinogenic ligands of C-type lectin receptors and RIG-I-like receptors were only obtained in the last few years, ${ }^{3,4}$ so there has not been enough time as yet to conduct comprehensive investigations between single nucleotide polymorphisms of genes encoding C-type lectin receptors and RIG-I-like receptors and cancer risk.

However, there is some evidence supporting the hypothesis that inherited features of C-type lectin receptor and RIG-I-like receptor structure can be associated with increased cancer risk.

\section{First premise: specific ligands}

Certain C-type lectin receptors and RIG-I-like receptors recognize PAMPs of oncogenic infectious agents. . $^{3,4,11,12}$

C-type lectin receptors:

- MRC1 (CD206, CLEC13D, mannose receptor) and PAMPs of Mycobacterium tuberculosis, Klebsiella pneumoniae, Streptococcus pneumoniae, Candida albicans, human immunodeficiency virus type-1 (HIV-1)

- CD207 (CLEC4K, langerin) and PAMPs of Candida spp, HIV-1

- LY75 (CD205, CLEC13B, DEC-205) and PAMPs of HIV-1

- CD209 (CLEC4L, DC-SIGN) and PAMPs of Mycobacterium spp, Schistosoma mansoni, C. albicans, HCV, HIV-1, cytomegalovirus

- CLEC7A (Dectin-1) and PAMPs of Mycobacterium spp

- CLEC1B (CLEC-2) and PAMPs of HIV-1

- CLEC6A (CLEC4N, Dectin-2) and PAMPs of $M$. tuberculosis, C. albicans, Paracoccidioides brasiliensis, Histoplasma capsulatum

- CLEC4E (Mincle) and PAMPs of M. tuberculosis and C. albicans

- CLEC4A (DCIR) and PAMPs of HIV-1 
RIG-I-like receptors:

- RIG-I and PAMPs of Epstein-Barr virus and hepatitis C virus

On the basis of known associations between inherited structural variations in Toll-like receptors and NOD-like receptors and cancer risk, ${ }^{1,2}$ and according to data about cancer types caused by carcinogenic infectious agents, ${ }^{11,12}$ it is possible to suggest that risk of lung cancer may be modulated by polymorphisms of the MRC1, CD209, CLEC7A, CLEC6A, and CLEC4E genes, oral cancer risk by single nucleotide polymorphisms of the $M R C 1, C D 207, C D 209$, CLEC6A, and CLEC4E genes, risk of glioblastoma and colorectal cancer by polymorphisms of the CD209 gene, hepatocellular carcinoma risk by polymorphisms of the CD209 and RIG-I genes, and risk of lymphoma, multiple myeloma, nasopharyngeal carcinoma, and esophageal and gastric cancer by single nucleotide polymorphisms of the $R I G-I$ gene. In addition, single nucleotide polymorphisms of MRC1, CD207, LY75, CD209, CLEC1B, and CLEC4A genes may correlate with cancer types associated with HIV-1 infection.

\section{Second premise: polymorphisms affecting function}

Certain polymorphisms of genes indicated above may have functional consequences on the molecular level that can lead to association of such single nucleotide polymorphisms with risk or progression of some diseases that may modulate cancer risk, so these gene polymorphisms may affect cancer risk indirectly. In addition, polymorphisms of these genes correlating with diseases that are not related to cancer risk may also be useful in oncogenomics because they may have functional consequences at the molecular level as well, although they have not been investigated in relation to association with cancer risk or progression.

For instance, it was suggested that variant alleles of MRC1 rs2477637, rs2253120, rs2477664, rs692527, rs1926736, and rs691005 gene polymorphisms are associated with development of asthma ${ }^{13}$ (eg, variant A allele of rs1926736 was connected with decreased asthma risk). In addition, Alter et al ${ }^{14}$ found that the variant A allele (S396) of rs1926736 (G396S) polymorphism is associated with a lower leprosy risk and, conversely, G allele (G396) correlates with increased risk of this disease. Interestingly, G396 did not influence leprosy risk in combination with T399 and L407 (amino acids resulting from variant alleles of rs2478577 and rs2437257, respectively). ${ }^{14}$ The authors noted that all three of these $\mathrm{MRCl}$ gene single nucleotide polymorphisms map to the second C-type lectin domain (CTLD2) of the MRC1 protein, with their in vitro results suggesting that a direct interaction between CTLD2 and an accessory receptor molecule is necessary in order for microbial ligand recognition to occur. ${ }^{14}$ It is logical to propose that such interaction would be sensitive to G396 only in the context of the A399-F407 haplotype, and not in the context of the T399-L407 haplotype. ${ }^{14}$ Thus, rs1926736 may have substantial functional consequences at the molecular level, but this depends on its relationship with other single nucleotide polymorphisms in the same exon. Finally, Hattori et a ${ }^{15}$ showed that a variant allele of rs691005 polymorphism, located within the $3^{\prime}$ untranslated region of the $M R C 1$ gene, is associated with a higher risk of sarcoidosis. Because of its location, it is feasible that this single nucleotide polymorphism may alter the regulatory binding sequence and influence mRNA expression. ${ }^{15}$

The only study investigating the association of polymorphisms of genes encoding C-type lectin receptors and RIG-I-like receptors with cancer risk is a study by Xu et al. ${ }^{16}$ They investigated single nucleotide polymorphisms of the CD209 gene and found that the GG genotype of the rs2287886, AA genotype of the -939 promoter polymorphism, and the G allele of the rs735239 single nucleotide polymorphism were connected with higher nasopharyngeal carcinoma risk. ${ }^{16}$ Polymorphisms in the promoter of the CD209 gene and in the CD209 gene were also associated with hemorrhage in patients with dengue fever ( $G$ allele of rs4804803), ${ }^{17,18}$ modulated tuberculosis risk ( $\mathrm{G}$ allele of rs4804803, A allele of rs735239), ${ }^{19-21}$ higher celiac disease risk in HLA-DQ2-negative cases ( $\mathrm{G}$ allele of rs4804803), ${ }^{22}$ increased ulcerative colitis risk in HLA-DR3-positive patients ( $\mathrm{G}$ allele of rs4804803) ${ }^{23}$ higher susceptibility to cytomegalovirus infection ( $\mathrm{G}$ allele of rs735240 and $\mathrm{C}$ allele of rs2287886), ${ }^{24}$ protection from lung cavitation ${ }^{20}$ and fever during tuberculosis ${ }^{25}$ (GG genotype and G allele of rs4804803), decreased HIV-1 infection risk (GG genotype of rs4804803), ${ }^{21}$ accelerated progression to acquired immune deficiency syndrome in HIV-1-infected hemophiliacs (C allele of rs2287886) ${ }^{26}$ decreased human T-lymphotropic virus type I infection risk ( $\mathrm{G}$ allele of rs4804803, A allele of rs2287886), ${ }^{27}$ increased severity of liver disease during hepatitis $\mathrm{C}$ virus infection ( $\mathrm{G}$ allele of rs 4804803$),{ }^{28}$ and better prognosis following severe acute respiratory syndrome ( $\mathrm{G}$ allele of rs4804803). ${ }^{29,30}$

It was shown that the A allele of the rs 4804803 single nucleotide polymorphism may increase gene expression in vitro, ${ }^{17}$ and, consequently, decreased CD209 gene 
Table I Results of case-control studies investigating the association of polymorphisms of genes encoding C-type lectin receptors, RIGI-like receptors, and proteins of their signaling pathways with various diseases, and conditions or features

\begin{tabular}{|c|c|c|c|c|}
\hline $\begin{array}{l}\text { Reference, } \\
\text { population }\end{array}$ & $\begin{array}{l}\text { SNP number, variant } \\
\text { allele frequency } \\
\text { in cases and controls }\end{array}$ & Disease or condition & Sample size & $\begin{array}{l}\text { OR and } 95 \% \mathrm{CI} \text { for } \\
\text { carriers of variant allele } \\
\text { (only positive or negative } \\
\text { statistically significant } \\
\text { results) }\end{array}$ \\
\hline
\end{tabular}

\section{mrcl}

Hattori et $\mathrm{al}^{13}$

Japanese,

Afro-American

populations

\author{
rs2477637 \\ Japanese 0.605-0.645, \\ Afro-American \\ 0.686-0.667) \\ rs2253120 \\ (Japanese 0.698-0.752, \\ Afro-American \\ 0.663-0.667) \\ rs247763। \\ (Japanese 0.484-0.522, \\ Afro-American \\ 0.238-0.267) \\ rs2477664 \\ Japanese 0.509-0.537, \\ Afro-American \\ 0.570-0.572) \\ rs692527 \\ Japanese 0.521-0.559, \\ Afro-American \\ $0.58 \mathrm{I}-0.389$ )
}

rs 1926736

Japanese 0.568-0.522,

Afro-American

0.855-0.86I)

rs691005

Japanese 0.705-0.679,

Afro-American

0.40I-0.26I)

Alter et al ${ }^{14}$

Vietnamese, Brazilian populations rs 1926736 (in Vietnamese controls 0.35, in Brazilian controls 0.32 )
Asthma

列

Leprosy

rs2437256 (in Vietnamese and Brazilian controls 0.21 ) rs2478577 (in Vietnamese controls 0 , in Brazilian controls 0.21 ) rs2437257 (in Vietnamese controls 0 , in Brazilian controls $0.2 \mathrm{I}$ )
Japanese,

446 cases, 424 controls;

Afro-American,

86 cases, 90 controls

Vietnamese, 704 cases, 396 controls; Brazilian, 384 cases, 399 controls
Japanese, dominant model 1.38 (1.02-1.87) Afro-

American, no association

Japanese, additive model I.34 (I.07-I.68); dominant model 1.55 (1.16-2.09); AfroAmerican, no association Japanese, no association; Afro-American, no association

Japanese, dominant model I.47 (I.06-2.05); Afro-

American, no association

Japanese, additive model I.25 (I.0I-I.55), dominant model I.39 (1.00-1.94); AfroAmerican, additive model 2.17 (1.40-3.37), dominant model 2.87 (I.43-5.80) recessive model 2.76 (1.34-5.70)

Japanese, additive model 0.76 (0.6I-0.95), recessive model $0.6 \mathrm{I}$ (0.4I-0.89) 0.6I; AfroAmerican, no association Japanese, no association; Afro-American, additive model I.8I (I.I6-2.8I), dominant model 2.43 (1.32-4.46)

Vietnamese, dominant model 0.76 (0.60-0.96), in the case with multibacillary leprosy, 0.7 I (0.5I-0.99); Brazilian, additive model, for carriers of wild-type G allele 1.34 (I.06-1.70), in the case with multibacillary leprosy, I.42 (I.05-1.93)

No association

No association

For carriers of wild-type $G$ allele, dominant model 0.75 (0.54-1.05); in the case with multibacillary leprosy 0.63 (0.4I-0.97) 
Table I (Continued)

\begin{tabular}{|c|c|c|c|c|}
\hline $\begin{array}{l}\text { Reference, } \\
\text { population }\end{array}$ & $\begin{array}{l}\text { SNP number, variant } \\
\text { allele frequency } \\
\text { in cases and controls }\end{array}$ & Disease or condition & Sample size & $\begin{array}{l}\text { OR and } 95 \% \mathrm{Cl} \text { for } \\
\text { carriers of variant allele } \\
\text { (only positive or negative } \\
\text { statistically significant } \\
\text { results) }\end{array}$ \\
\hline \multirow[t]{9}{*}{$\begin{array}{l}\text { Hattori et al }{ }^{15} \\
\text { Japanese population }\end{array}$} & $\begin{array}{l}\text { rs } 2477637(0.4 \mathrm{I} 2-0.355 \\
\text { AG genotype } 0.37-0.44 \mathrm{I} \\
\text { GG genotype } 0.227-0.134)\end{array}$ & Sarcoidosis & I8I cases, 424 controls & No association \\
\hline & $\begin{array}{l}\text { rs } 2253 \mathrm{I} 20(0.30 \mathrm{I}-0.248, \\
\text { AG genotype } 0.448-0.325, \\
\text { GG genotype } 0.077-0.085)\end{array}$ & & & No association \\
\hline & $\begin{array}{l}\text { rs } 247763 \mathrm{I}(0.472-0.478, \\
\text { AC genotype } 0.547-0.479 \\
\text { CC genotype } 0.199-0.238)\end{array}$ & & & No association \\
\hline & $\begin{array}{l}\text { rs } 2477664(0.472-0.463, \\
\text { AT genotype } 0.514-0.455 \text {, } \\
\text { TT genotype } 0.215-0.236)\end{array}$ & & & No association \\
\hline & $\begin{array}{l}\text { rs692527 }(0.492-0.44 \mathrm{I} \\
\text { AG genotype } 0.464-0.505, \\
\text { GG genotype } 0.26-0.189)\end{array}$ & & & No association \\
\hline & $\begin{array}{l}\text { rs I } 926736(0.453-0.478, \\
\text { AG genotype } 0.475-0.521 \text {, } \\
\text { AA genotype } 0.215-0.217)\end{array}$ & & & No association \\
\hline & $\begin{array}{l}\text { rs544995 }(0.298-0.295, \\
\text { AG genotype } 0.43 \mathrm{I}-0.467 \text {, } \\
\text { AA genotype } 0.083-0.06 \mathrm{I})\end{array}$ & & & No association \\
\hline & $\begin{array}{l}\text { rs5543I3 }(0.34-0.396, \\
\text { AG genotype } 0.492-0.462, \\
\text { AA genotype } 0.094-0.153)\end{array}$ & & & No association \\
\hline & $\begin{array}{l}\text { rs69I005 }(0.376-0.321 \\
\text { TC genotype } 0.376-0.458, \\
\text { CC genotype } 0.188-0.092)\end{array}$ & & & $\begin{array}{l}\text { Recessive model, } 2.53 \\
(1.47-4.37)\end{array}$ \\
\hline \multicolumn{5}{|l|}{ CD209 } \\
\hline \multirow[t]{6}{*}{ Cantonese population } & $\begin{array}{l}\text {-116 promoter } \\
\text { polymorphism } \\
\text { (0.006 in controls) }\end{array}$ & NPC & 444 cases, 464 controls & No association \\
\hline & $\begin{array}{l}\text { rs } 2287886 \\
(0.275 \text { in controls })\end{array}$ & & & $\begin{array}{l}\text { I.42 (I.I5-I.74); for } \\
\text { carriers of AG genotype, } \\
\text { I.4I (I.05-I.88), for } \\
\text { carriers of GG genotype, } \\
2.10(1.23-3.59)\end{array}$ \\
\hline & $\begin{array}{l}\text {-190 promoter } \\
\text { polymorphism } \\
\text { (in controls } 0.003 \text { ) }\end{array}$ & & & No association \\
\hline & $\begin{array}{l}\text { rs } 4804803 \\
\text { (in controls } 0.085 \text { ) }\end{array}$ & & & No association \\
\hline & $\begin{array}{l}\text { rs735239 } \\
\text { (in controls } 0.154 \text { ) }\end{array}$ & & & $\begin{array}{l}\text { I.47 (I.I4-I.90); for carriers } \\
\text { of AG genotype, I. } 44 \\
(1.05-1.98)\end{array}$ \\
\hline & $\begin{array}{l}\text { rs735240 } \\
\text { (in controls } 0.222 \text { ) }\end{array}$ & & & $\begin{array}{l}\text { I. } 43 \text { (I.I5-I.79); for carriers } \\
\text { of AA genotype, } 2.52 \\
(1.29-4.93)\end{array}$ \\
\hline $\begin{array}{l}\text { Sakuntabhai et al }{ }^{17} \\
\text { Thai population }\end{array}$ & $\begin{array}{l}\text { rs } 4804803 \text { ( } 0.093 \\
\text { in dengue disease patients, } \\
0.023 \text { in dengue fever } \\
\text { patients, } 0.116 \text { in dengue } \\
\text { hemorrhagic fever patients, } \\
0.104 \text { in controls) }\end{array}$ & $\begin{array}{l}\text { Dengue disease, } \\
\text { dengue fever, dengue } \\
\text { hemorrhagic fever }\end{array}$ & 606 cases, 696 controls & $\begin{array}{l}\text { Risk of hemorrhage during } \\
\text { dengue fever, } 5.84 \\
(2.77-12.31) \text {; risk of dengue } \\
\text { fever, } 0.204 \text {; decreased } \\
\text { CD209 gene expression }\end{array}$ \\
\hline
\end{tabular}


Table I (Continued)

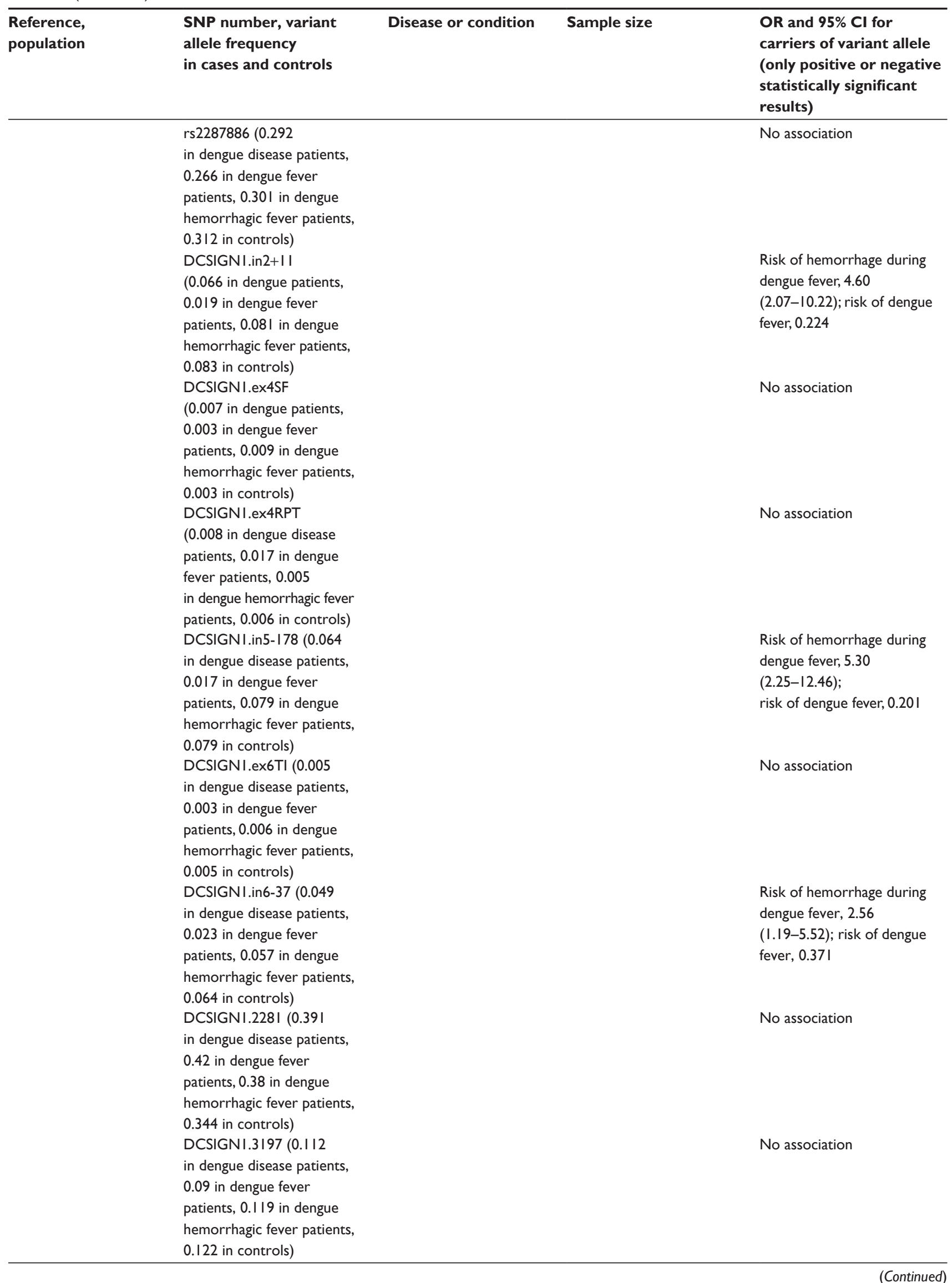


Table I (Continued)

\begin{tabular}{|c|c|c|c|c|}
\hline $\begin{array}{l}\text { Reference, } \\
\text { population }\end{array}$ & $\begin{array}{l}\text { SNP number, variant } \\
\text { allele frequency } \\
\text { in cases and controls }\end{array}$ & Disease or condition & Sample size & $\begin{array}{l}\text { OR and } 95 \% \mathrm{Cl} \text { for } \\
\text { carriers of variant allele } \\
\text { (only positive or negative } \\
\text { statistically significant } \\
\text { results) }\end{array}$ \\
\hline & $\begin{array}{l}\text { DCSIGNI.3852 ( } 0.243 \\
\text { in dengue disease patients, } \\
0.24 \text { in dengue fever } \\
\text { patients, } 0.244 \text { in dengue } \\
\text { hemorrhagic fever patients, } \\
0.267 \text { in controls) }\end{array}$ & & & No association \\
\hline $\begin{array}{l}\text { Wang et al }{ }^{18} \\
\text { Taiwanese population }\end{array}$ & $\begin{array}{l}\text { rs } 4804803 \text { ( } 0.084 \\
\text { in dengue patients, } 0.054 \\
\text { in dengue fever patients, } \\
0.122 \text { in dengue hemorrhagic } \\
\text { fever cases, } 0.028 \text { in other } \\
\text { non-dengue febrile illness } \\
\text { cases, } 0.038 \text { in controls) }\end{array}$ & $\begin{array}{l}\text { Dengue disease, } \\
\text { dengue fever, dengue } \\
\text { hemorrhagic fever }\end{array}$ & $\begin{array}{l}\text { I } 76 \text { dengue fever cases, } \\
\text { I } 35 \text { dengue hemorrhagic } \\
\text { fever cases, I } 43 \text { patients } \\
\text { with other non-dengue } \\
\text { febrile illnesses, } \\
\text { I } 20 \text { controls }\end{array}$ & $\begin{array}{l}\text { Risk of dengue infection, } \\
2.34 \text { (I.I4-4.83); risk of } \\
\text { dengue hemorrhagic fever, } \\
3.57 \text { ( I.67-7.63); risk of } \\
\text { hemorrhage during dengue } \\
\text { fever, } 2.44 \text { (I.36-4.40); } \\
\text { increased levels of TNF- } \alpha \text {, } \\
\text { IL-I } 2 \text { p40, IP-10 }\end{array}$ \\
\hline \multirow[t]{29}{*}{$\begin{array}{l}\text { Barreiro et al }{ }^{19} \\
\text { South African } \\
\text { population }\end{array}$} & rs2048022 (0,434-0.483) & Tuberculosis & $35 \mathrm{I}$ cases, 360 controls & No association \\
\hline & rs I $380229(0.36 \mathrm{I}-0.384)$ & & & No association \\
\hline & rs650389 (0.152-0.195) & & & No association \\
\hline & rs870384 (0.483-0.468) & & & No association \\
\hline & rs695982 (0.32I-0.277) & & & No association \\
\hline & rs708682 (0.1 18-0.123) & & & No association \\
\hline & rs715774 (0.143-0.16I) & & & No association \\
\hline & rs|433456 (0.199-0.197) & & & No association \\
\hline & rs807|3। (0.355-0.339) & & & No association \\
\hline & rsII672183 (0.12-0.117) & & & No association \\
\hline & rs2024628 (0.422-0.465) & & & No association \\
\hline & rsI028I84 (0.342-0.39) & & & No association \\
\hline & rs2056773 (0.395-0.37I) & & & No association \\
\hline & rs|479067 (0.259-0.284) & & & No association \\
\hline & rs327747 (0.258-0.292) & & & No association \\
\hline & rs I266532I (0.142-0.129) & & & No association \\
\hline & rs I $566838(0.465-0.458)$ & & & No association \\
\hline & rs I $2785524(0.39-0.424)$ & & & No association \\
\hline & rs975423 (0.35I-0.378) & & & No association \\
\hline & rs914904 (0.292-0.282) & & & No association \\
\hline & rs876287 (0.4I3-0.409) & & & No association \\
\hline & rsI582598 (0.275-0.265) & & & No association \\
\hline & rs|364| $98(0.252-0.227)$ & & & No association \\
\hline & rs739259 (0.36I-0.39) & & & No association \\
\hline & rsI69479 (0.133-0.1I5) & & & No association \\
\hline & rs4804803 (0.454-0.402) & & & $\mathrm{I} .48(1.08-2.02)$ \\
\hline & rs735239 (0.089-0.14I) & & & $\begin{array}{l}\text { For carriers of A allele, } 1.85 \\
(1.29-2.66)\end{array}$ \\
\hline & rs735240 (0.283-0.3।3) & & & No association \\
\hline & rs2287886 (0.27I-0.288) & & & No association \\
\hline Vannberg et $\mathrm{al}^{20}$ & rs4804803 (in Gambian & Tuberculosis & Gambian: 678 cases, & For Gambian population, \\
\hline Gambian, Guinean, & population $0.48-0.54$ & & 327 controls Guinean: & 0.75 (0.6I-0.94); overall, 0.86 \\
\hline Guinea-Bissauan, & in Guinean population & & 151 cases, 180 controls & (0.77-0.96); for cavitating \\
\hline \multirow[t]{4}{*}{ Malawian populations } & $0.489-0.47$, in Guinea- & & Guinea-Bissauan: & tuberculosis, $0.42(0.27-0.65)$ \\
\hline & Bissauan population & & I62 cases, $14 \mid$ controls & \\
\hline & $0.475-0.504$, in Malawian & & Malawian: 244 cases, & \\
\hline & population $0.352-0.364$ ) & & 295 controls & \\
\hline
\end{tabular}


Table I (Continued)

\begin{tabular}{|c|c|c|c|c|}
\hline $\begin{array}{l}\text { Reference, } \\
\text { population }\end{array}$ & $\begin{array}{l}\text { SNP number, variant } \\
\text { allele frequency } \\
\text { in cases and controls }\end{array}$ & Disease or condition & Sample size & $\begin{array}{l}\text { OR and } 95 \% \mathrm{CI} \text { for } \\
\text { carriers of variant allele } \\
\text { (only positive or negative } \\
\text { statistically significant } \\
\text { results) }\end{array}$ \\
\hline \multirow[t]{4}{*}{$\begin{array}{l}\text { Selvaraj et } \mathrm{al}^{2 !} \\
\text { South Indian } \\
\text { population }\end{array}$} & rs4804803 (0.18I-0.223) & Tuberculosis, HIV & $\begin{array}{l}238 \text { HIV cases, } \\
\text { I07 HIV+ and } \\
\text { tuberculosis cases, } \\
\text { I } 57 \text { controls }\end{array}$ & $\begin{array}{l}\text { For carriers of GG genotype, } \\
\text { risk of tuberculosis among } \\
\text { HIV-infected patients, } 9.8 \\
(2.2-44.3)\end{array}$ \\
\hline & rs2287886 (0.47I-0.468) & & & No association \\
\hline & rs7252229 (0.105-0.10I) & & & No association \\
\hline & rsI544767 (0.105-0.108) & & & No association \\
\hline $\begin{array}{l}\text { Nunez et } \mathrm{al}^{22} \\
\text { Spanish population }\end{array}$ & rs4804803 $(0.23-0.21)$ & Celiac disease & 103 cases, 312 controls & $\begin{array}{l}\text { For carriers of GG genotype; } \\
\text { for HLA-DQ2(-)-individuals } \\
\text { compared with HLA-DQ2(+) } \\
\text { individuals and controls, } 3.73 \\
(\text { I.18-1I.03) }\end{array}$ \\
\hline $\begin{array}{l}\text { Nunez et a }{ }^{23} \\
\text { Spanish population }\end{array}$ & $\begin{array}{l}\text { rs } 4804803 \text { ( } 0.25 \text { in Crohn's } \\
\text { disease patients, } 0.22 \\
\text { in ulcerative colitis patients, } \\
0.22 \text { in controls) }\end{array}$ & $\begin{array}{l}\text { Crohn's disease, } \\
\text { ulcerative colitis }\end{array}$ & $\begin{array}{l}515 \text { Crohn's disease } \\
\text { cases, } 497 \text { ulcerative } \\
\text { colitis cases, } 73 \text { I controls }\end{array}$ & $\begin{array}{l}\text { Risk of ulcerative colitis in } \\
\text { HLA-DR3-positive patients } \\
\text { I.77 (I.04-3.02) }\end{array}$ \\
\hline
\end{tabular}

Mezger et al ${ }^{24}$

European population
Human CMV reactivation and disease after allogeneic stem cell transplantation

rs735240

rs4804803 (0.06I-0.073)

rs735239 (0.207-0.234)

rs2287886

rs4804803

rs2287886 (0.594-0.795)

Kashima et $\mathrm{a}^{27}$

Mixed population

from different

continents

\author{
-20I promoter \\ polymorphism (0.038-0.016) \\ -332 promoter \\ polymorphism (0.03-0) \\ rs4804803 (0.144-0.297)
}

rs4804803 (0.25-0.19)

rs4804803
Tuberculosis

AIDS progression

HLTV-I-infection

66 cases, 33 controls

104 HIV-I-positive
70 patients with human CMV reactivation, 59 patients with human CMV disease, 65 controls

237 cases, 244 controls Japanese hemophiliacs

|3| cases, 79 controls

585 cases with lower LDH level, 96 cases with higher LDH level
Risk of human CMV disease, I.88 (0.9|-3.87)

Risk of human CMV reactivation, 2.4I (I.22-4.75); risk of human CMV disease,

2.01 ( $1.05-3.86)$

$0.209(0.058-0.758)$

No association

Risk of accelerated

AIDS progression:

I.95 (1.039-3.677)

No association

For carriers of $A$ allele: Risk of HTLV-I-infection: 0.3758

(0.1954-0.7229)

For carriers of AA genotype: Risk of HTLV-I-infection: 0.1 I I 6 (0.02 I 68-0.5745)

No association

No association

For carriers of $A$ allele: Risk of HTLV-I-infection: 2.5 I I

(1.218-5.179)

Increased risk of advanced liver disease

Risk of higher LDH level during SARS, 0.4I (0.20-0.86); decreased expression of CD209 gene; SpI and AP2 proteins bind more effectively to $\mathrm{G}$ allele of rs 4804803 
Table I (Continued)

\begin{tabular}{llll}
\hline $\begin{array}{l}\text { Reference, } \\
\text { population }\end{array}$ & $\begin{array}{l}\text { SNP number, variant } \\
\text { allele frequency }\end{array}$ & Disease or condition & Sample size
\end{tabular}

in cases and controls

rs4804803

Chan et a ${ }^{30}$

Hong Kong

population

CLEC7A (Dectin-I)

Plantinga et al ${ }^{31}$

Dutch population

Cunha et $\mathrm{al}^{32}$

Italian population

Chai et a $^{33}$

Dutch and Flemish population rs 16910526 (0.19 in patients without hematopoietic transplantation with invasive aspergillosis, 0.077

in controls, 0.07 in patients with transplantation

[with and without invasive aspergillosis])

\section{Invasive aspergillosis}

Invasive aspergillosis

7I cases with invasive aspergillosis after hematopoietic stem cell transplantation, $2 \mathrm{I}$ cases with invasive aspergillosis without transplantation, 108 controls with transplantation
205 cases

with hematopoietic

stem cell transplantation
OR and $95 \% \mathrm{Cl}$ for carriers of variant allele (only positive or negative statistically significant results)

Risk of higher LDH level during SARS, 0.4I (0.20-0.86)

Diminished TNF- $\alpha$ and IL-I $\beta$ production in cells from homozygous and heterozygous individuals; the TLR2/Dectin-I synergism was reduced in cells isolated from heterozygous and homozygous subjects Risk of invasive aspergillosis after hematopoietic stem cell transplantation, polymorphic donor + wild-type recipient, 2.50 (1.00-6.53) Polymorphic donor + polymorphic recipient: 3.89 (I.5I-9.99) Unstimulated CDI4positive monocytes from polymorphic persons display a decreased surface expression of Dectin I in response to $\beta$-glucan or A conidia, PBMCs from heterozygous persons showed decreased production of IL-I $\beta$, IL-6, IL-I0, IL-I7A, and IFN- $\gamma$ Increased risk of invasive aspergillosis in patients without hematopoietic transplantation PBMCs from variant homozygous persons had reduced proinflammatory TNF- $\alpha$ and IL-6 production in response to heat-killed Aspergillus fumigatus hyphae, Candida albicans blastoconidia, and live A. fumigatus conidia; monocytederived macrophages from polymorphic individuals had deficient expression of the Dectin I receptor; stimulation using $\beta$-glucan failed to generate a TNF- $\alpha$ response in the Dectin I-deficient monocyte-derived macrophages from variant homozygotes

(Continued) 
Table I (Continued)

\begin{tabular}{|c|c|c|c|}
\hline $\begin{array}{l}\text { Reference, } \\
\text { population }\end{array}$ & $\begin{array}{l}\text { SNP number, variant } \\
\text { allele frequency } \\
\text { in cases and controls }\end{array}$ & Disease or condition & Sample size \\
\hline Plantinga et $\mathrm{al}^{34}$ & rsI6910526 (0.106-0.138) & Colonization with & 142 cases \\
\hline Dutch population & & Candida spp & $\begin{array}{l}\text { with hematopoietic } \\
\text { stem cell transplantation, } \\
\text { I } 38 \text { controls }\end{array}$ \\
\hline
\end{tabular}

OR and $95 \% \mathrm{Cl}$ for carriers of variant allele (only positive or negative statistically significant results)

Risk of Candida spp

colonization, 12.0

(2.5-57.I); risk of Candida spp colonization after transplantation, 15.5 (1.9-125.6); monocytes from the variant homozygous individuals exhibited no Dectin I expression on the cell surface, whereas cells from heterozygous individuals had intermediate cell surface expression; IL-I $\beta$ induction by $C$. albicans was lower in cells from individuals bearing the polymorphism; no possibility to amplify TLR2 signaling by Dectin I in cells isolated from variant homozygous individuals

Plantinga et al ${ }^{35}$

East African

population

RIG-I

Ovsyannikova et $\mathrm{al}^{38}$ rs $|08| 382 \mid$

US population

Ovsyannikova et $\mathrm{al}^{39}$ US population

rs669260

Hu et $\mathrm{a}^{40}$ rs $1081383 \mid$ US population

rs9650702

rs626214

rs5925I5

rs6476363

rs3739674

rs 10813829

rs4633। 44

rs3824456

rs 10813831

rs $108|383|$

uspution
Oropharyngeal candidiasis

Cytokine immune response in healthy children following rubella vaccination

children follov
225 cases with HIV

738 cases

IFN- $\gamma$ production capacity and ability to bind zymosan was markedly lower in cells from subjects bearing the polymorphism

Increased level of IFN- $\gamma$

Increased level of IFN- $\gamma$ and decreased level of GM-CSF Increased level of IFN- $\gamma$ Increased level of IFN- $\gamma$ and TNF- $\alpha$

Decreased level of TNF- $\alpha$ Decreased level of TNF- $\alpha$ Decreased level of TNF- $\alpha$ Decreased level of TNF- $\alpha$ Increased level of TNF- $\alpha$ Decreased level of GM-CSF and IL-6

Decreased rubella-specific antibody response (median antibody level)

rubella vaccination

738 cases

Increased rubella-specific antibody response Increased gene expression in Newcastle disease virusinfected cells No association
Cytokine immune $\quad 130$ cases response to Newcastle disease 
Table I (Continued)

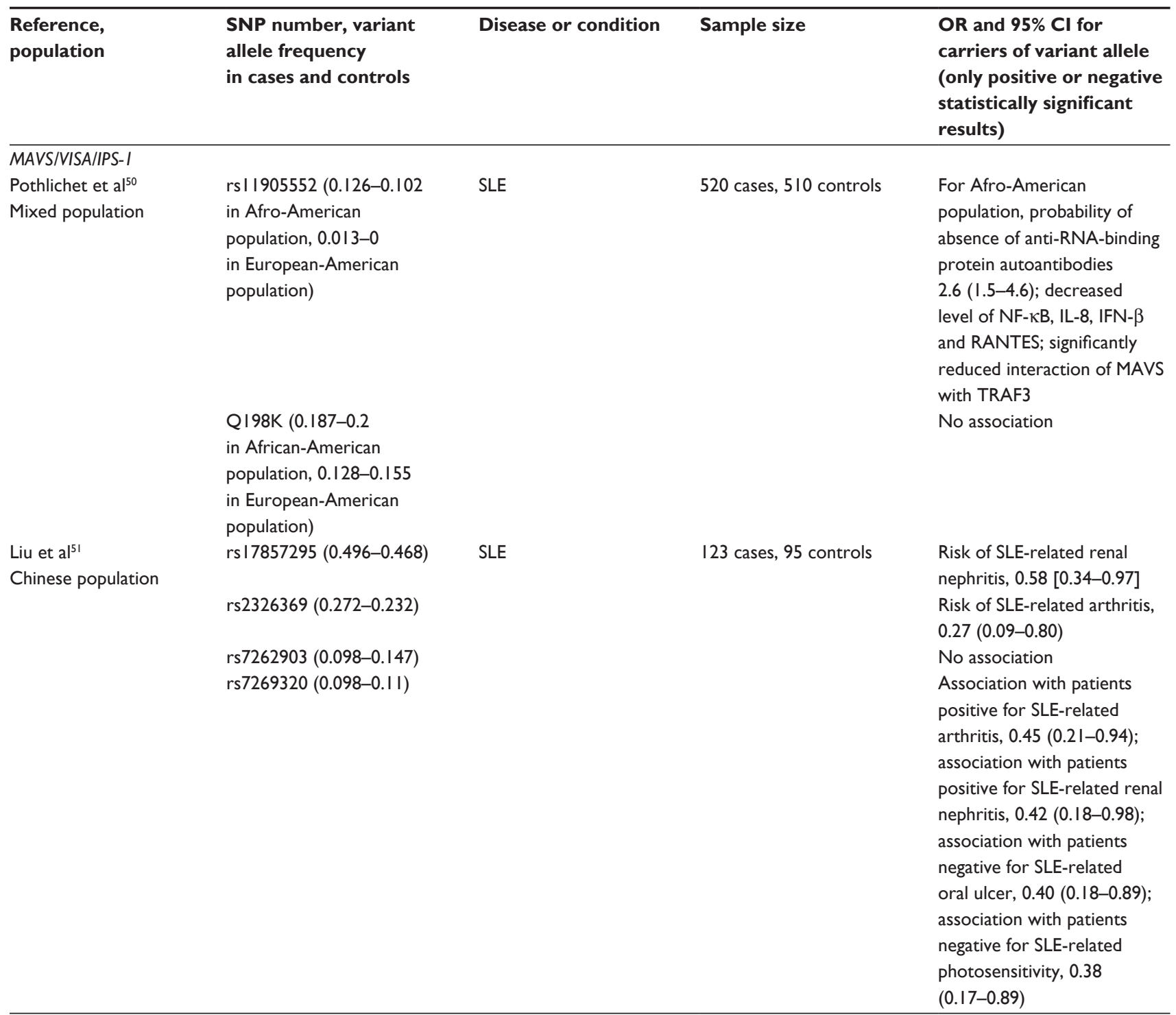

Abbreviations: SNP, single nucleotide polymorphism; OR, odds ratio; $\mathrm{Cl}$, confidence interval; $\mathrm{MRC}$, mannose receptor $\mathrm{C}$; $\mathrm{CD}$, cluster of differentiation; NPC, nasopharyngeal carcinoma; DC-SIGN, dendritic cell-specific intercellular adhesion molecule-3-grabbing non-integrin; TNF, tumor necrosis factor; IL, interleukin; IP, interferon-gamma inducible protein 10; HIV, human immunodeficiency virus; AIDS, acquired immunodeficiency virus; HTLV, human T-cell lymphotropic virus; HCV, hepatitis C virus; SARS, severe acute respiratory syndrome; LDH, lactate dehydrogenase; Sp, specificity protein; AP, activator protein; CLEC, C-type lectin domain, the next number is the family number 7, the next letter is the letter of family member; TLR, toll-like receptor; PBMC, peripheral blood mononuclear cell; IFN, interferon; RIG-I, retinoic acid-inducible gene I; GM-CSF, granulocyte-macrophage colony-stimulating factor; MAVS/VISA/IPS-I, mitochondrial antiviral signaling protein/virus-induced signaling adapter/induced by phosphate starvation-I; NF-KB, necrosis factor kappa B; RANTES, regulated on activation, normal T-cell expressed and secreted; TRAF, TNF receptor-associated factor; SLE, systemic lupus erythematosus.

expression in subjects with the $\mathrm{G}$ allele may result in an impaired immune response against hepatitis $\mathrm{C}$ virus, ${ }^{28}$ M. tuberculosis, ${ }^{19,21}$ and bacteria potentially causing celiac disease $^{22}$ and ulcerative colitis, ${ }^{23}$ that elevates the risk of diseases caused by these infectious agents. Such a decreased immune response may protect from hemorrhage during dengue fever, ${ }^{17}$ from lung cavitation, ${ }^{20}$ from fever during tuberculosis, ${ }^{25}$ and from lung injury during severe acute respiratory syndrome $^{29,30}$ as a result of less cytokine production and diminished activation of immune cells. However, from the point of view of Vannberg et al, ${ }^{20}$ conversely, lower CD209 gene expression as a consequence of $\mathrm{G}$ allele of rs4804803 polymorphism may protect against tuberculosis because of decreased production of proinflammatory cytokines such as interleukin-4. Further fundamental, translational, and clinical studies are necessary to clarify these discrepancies. Nevertheless, although there are a number of reasons for the discrepancies between studies devoted to the association between CD209 single nucleotide polymorphisms and development of tuberculosis, but confounding host, bacterial, and 
environmental factors between different study populations should be taken into account. In addition, Mezger et $\mathrm{al}^{24}$ demonstrated that alleles of rs735240 and rs2287886 polymorphisms may also influence CD209 gene expression and thus affect transcription factor binding.

In relation to the $C L E C 7 A$ (Dectin-1) gene, it was also found that a variant allele of rs16910526 polymorphism is associated with impaired cytokine production by macrophages $^{31,32}$ and with a defective response to Aspergillus and Candida invasion. ${ }^{33,34}$ The variant $\mathrm{S}$ form of $\mathrm{I} 223 \mathrm{~S}$ polymorphism was characterized by a lower capacity of the receptor to bind zymosan. ${ }^{35}$

Among polymorphisms of genes encoding RIG-Ilike receptors, $R I G-I$ single nucleotide polymorphisms are the most investigated. Pothlichet et $\mathrm{al}^{36}$ conducted a comprehensive study investigating the functional consequences of rs36055726 (P229fs) and rs11795404 (S183I) polymorphisms. They found that the variant allele of rs36055726 results in a truncated constitutively active RIG-I (that leads to permanent production of proinflammatory mediators, particularly antiviral), and, conversely, the variant allele of rs11795404 induces an abortive conformation of RIG-I, causing formation of unintended stable complexes between CARD modules of RIG-I and between RIG-I and its downstream adapter protein, MAVS, rendering RIG-I incapable of downstream signaling and further cytokine synthesis. ${ }^{36}$ Moreover, Shigemoto et al identified a variant of rs11795404 as a loss-of-function allele. ${ }^{37}$ Ovsyannikova et $\mathrm{al}^{38,39}$ showed that a minor allele of rs10813831 polymorphism is associated with a decrease in the rubella virus-specific granulocyte-macrophage colony-stimulating factor/interleukin-6/IgG response, whilst a variant allele of rs3824456 is connected with an increase in the rubella virusspecific tumor necrosis factor alpha response, and a variant allele of rs669260 correlates with an increase in the rubellaspecific antibody level. Hu et $\mathrm{al}^{40}$ discovered that a variant allele of rs10813831 polymorphism leads to increased gene expression and, consequently, cytokine production due to an amino acid substitution in the CARD domain of RIG-I that results in functional alteration of this RIG-I-like receptor.

There are also a lot of studies investigating the role of IFIH1/MDA5 (the gene encoding MDA5 protein that is also a RIG-I-like receptor) single nucleotide polymorphisms in the etiology of autoimmune diseases, but almost all of them are devoted to type 1 diabetes and multiple sclerosis, and data about the association of these diseases with cancer risk are conflicting, in that some studies showed an increased risk in patients with type 1 diabetes and multiple sclerosis, ${ }^{41,42}$ and
Table 2 Polymorphisms of genes encoding C-type lectin receptors, RIG-I-like receptors, and proteins of their specific signaling pathways that have known functional consequences and may be relevant to oncogenomics

\begin{tabular}{|c|c|}
\hline Gene & Single nucleotide polymorphism \\
\hline \multicolumn{2}{|c|}{ Genes encoding CLRs } \\
\hline \multirow[t]{8}{*}{$\mathrm{MRCl}$} & rs1926736* \\
\hline & rs2478577* \\
\hline & rs2437257* \\
\hline & rs691005* \\
\hline & rs2477664 \\
\hline & rs692527 \\
\hline & rs2253120 \\
\hline & rs2477637 \\
\hline \multirow[t]{4}{*}{ CD209 } & rs2287886* \\
\hline & rs735239* \\
\hline & rs4804803* \\
\hline & rs735240* \\
\hline \multirow[t]{2}{*}{ CLEC7A } & rsI6910526* \\
\hline & $1223 \mathrm{~S}$ \\
\hline \multicolumn{2}{|c|}{ Genes encoding RLRs } \\
\hline \multirow[t]{13}{*}{ RIG-I } & rs36055726* \\
\hline & rsII795404* \\
\hline & rsl08I383I* \\
\hline & rs3824456 \\
\hline & rs669260 \\
\hline & rs9650702 \\
\hline & rs626214 \\
\hline & rs5925I5 \\
\hline & rs6476363 \\
\hline & rs3739674 \\
\hline & rs 10813829 \\
\hline & rs4633I44 \\
\hline & rs $108|382|$ \\
\hline \multicolumn{2}{|c|}{ Genes encoding proteins of CLR and RLR intracellular signaling pathways } \\
\hline \multirow[t]{4}{*}{ MAVS/VISAIIPS-I } & rsII905552 \\
\hline & rs 17857295 \\
\hline & rs 2326369 \\
\hline & rs7269320 \\
\hline
\end{tabular}

Note: *Single nucleotide polymorphisms that can be valued as the most promising for further oncogenomic investigation.

Abbreviations: PRRs, pattern recognition receptors; PAMPs, pathogen-associated molecular patterns; DAMPs, damage-associated molecular patterns; TLRs, Toll-like receptors; NLRs, NOD-like receptors; CLRs, C-type lectin receptors; RLRs, RIG-I-like receptors; SNPs, single nucleotide polymorphisms; MRC, mannose receptor C; $C D$, cluster of differentiation; CLEC, C-type lectin domain, the next number is the family number 7 , the next letter is the letter of family member; HIV, human immunodeficiency virus; LY, lymphocyte antigen; DEC-205, dendritic and epithelial cells $205 \mathrm{kDa}$; DC-SIGN, dendritic cell-specific intercellular adhesion molecule-3-grabbing non-integrin; HCV, hepatitis C virus; CMV, cytomegalovirus; DCIR, dendritic cell immunoreceptor; RIG-I, retinoic acid-inducible gene I; EBV, Epstein-Barr virus; CARD, caspase recruitment domain; MAVS, mitochondrial antiviral signaling protein; IFIH, interferon induced with helicase $\mathrm{C}$ domain; MDA, melanoma differentiation-associated gene; VISA, virus-induced signaling adapter; IPS-I, induced by phosphate starvation-I.

in other investigations no connection or decreased risk of cancer has been observed. ${ }^{43-49}$ Taking into account that there are no carcinogenic infectious agents recognizing MDA5, it does not seem to be prudent to investigate $I F I H 1 / M D A 5$ gene polymorphisms from the oncogenomic point of view. 
In addition, polymorphisms of genes coding for components of the Toll-like receptor signaling pathway may modulate cancer risk as single nucleotide polymorphisms of the TLR gene family. ${ }^{1}$ The same statement can be true for C-type lectin receptor and RIG-I-like receptor signaling pathways. For instance, a variant allele of rs1 1905552, encoding MAVS/ VISA/IPS-1, a key downstream signaling molecule of RIG-I and MDA5, was associated with a particular systemic lupus erythematosus phenotype.$^{50}$ It was found that this single nucleotide polymorphism leads to reduced production of type I interferon and other proinflammatory mediators, and also to the absence of anti-RNA-binding protein autoantibodies. ${ }^{50}$ In addition, variant alleles of rs17857295 and rs2326369 polymorphisms of the MAVS/VISA/IPS-1 gene were associated with nephritis and arthritis in patients suffering from systemic lupus erythematosus. ${ }^{51} \mathrm{~A}$ variant allele of another single nucleotide polymorphism of this gene, rs7269320, showed associations with different clinical characteristics of this autoimmune disease.$^{51}$ All the population case-control studies mentioned above are summarized in Table 1.

\section{Conclusion and future directions}

All polymorphisms of genes encoding C-type lectin receptors, RIG-I-like receptors, and proteins of their specific signaling pathways that have known functional consequences and may be relevant to oncogenomics are summarized in Table 2. The fundamental basis for the association of the inherited coding variation in genes encoding C-type lectin receptors and RIG-I-like receptors with cancer is represented by the defects in the immune response (that are caused by various single nucleotide polymorphisms) against specific carcinogenic infectious agents. Some polymorphisms may be valued as the most promising for further oncogenomic investigations on the basis of their association with cancer risk or because of their substantial functional consequences on the molecular level according to the following concept:

Gene polymorphism may be included on the short list for further oncogenomic studies if:

- The single nucleotide polymorphism leads to substantial functional consequences at the molecular level (for instance, it strongly affects transcription, splicing, translation, stability and transport of pre-mRNA, mRNA, noncoding RNA, or protein encoding by the gene, or it noticeably influences signaling of synthesized protein)

- It is associated with risk of cancer in population studies

- It has functional consequences at the molecular level and it is strongly associated with a condition that significantly increases the risk of cancer (threshold may vary for each cancer type)

The gene polymorphism can be also included on the extended list if:

- It is characterized by more subtle functional alterations in a gene that, nonetheless, result in qualitative or quantitative alterations of the encoding protein (or noncoding RNA)

- It is associated with a condition that substantially increases the risk of cancer but has not specifically been identified to increase the risk of cancer.

According to this concept, the indicated short list of polymorphisms includes rs1926736, rs2478577, rs2437257, rs691005 (all located in the $M R C 1$ gene), rs2287886, -939 promoter polymorphism, rs735239, rs735240, rs4804803 (all located in the CD209 gene), rs16910526 (CLEC7A gene), and rs36055726, rs11795404, rs10813831 (all located in the RIG-I gene). Other polymorphisms mentioned in this article may be added to the extended list for further investigations. Polymorphisms with known functional effects (rs1926736, rs2437257, rs691005, rs2287886, rs735240, rs4804803, rs16910526) were associated with relatively significant modulation of risk of diseases (as shown in Table 1) which is logical and demonstrates the correctness of the studies in which functional consequences of such single nucleotide polymorphisms were analyzed. There are still no comprehensive functional investigations for other single nucleotide polymorphisms correlated with risk of disease, so it is difficult to conclude which of them have independent significance, and which of them are just in linkage disequilibrium with truly functional variants.

In addition, PAMPs of specific infectious agents recognized by each C-type lectin receptor or RIG-I-like receptor define cancer types which can be primarily associated with inherited structural variation in the receptors discussed earlier. Furthermore, if a single nucleotide polymorphism of a gene encoding a specific C-type lectin receptor or RIGI-like receptor is associated with risk or progression features of certain malignancies, polymorphisms in genes encoding specific signaling molecules constituting pathways of these receptors should correlate with similar neoplasms, if they have substantial functional consequences at the molecular level. The issue of an association of single nucleotide polymorphisms of genes encoding C-type lectin receptors, RIGI-like receptors, and proteins of pattern recognition receptor pathways with various features of cancer progression is open, and only further population studies would be likely to give a definite answer. 
Reasons for discrepancies in different investigations analyzing the association of polymorphisms in genes encoding C-type lectin receptors, RIG-I-like receptors, and the proteins of their signaling pathways with various aspects of cancer development may include confounding host, bacterial, or environmental factors in different ethnicities modulating penetrance of variant alleles and affecting the risk of conditions increasing cancer risk (such as autoimmune diseases, precancerous gastric lesions, tuberculosis, recurrent pneumonia), different bacterial impact on the etiology of such conditions in different populations (that will be reflected in different features of C-type lectin receptor/ RIG-I-like receptor-mediated immune response because of specific C-type lectin receptor/RIG-I-like receptor-ligand interaction), differences in sample size, in clinicopathological characteristics between study samples, in prevalence of infectious agents in case and control groups, diagnostics, stratification, genotyping methods, and chance.

Another interesting issue is that associations between single nucleotide polymorphisms of genes encoding C-type lectin receptors and RIG-I-like receptors and cancer risk can be skewed by differences between cohorts in various immune responses and infections that may not influence cancer development. The problem is that the design in an epidemiological study having a large sample is very seldom ideal. Stratification by status of chronic infection is rather difficult because of their extreme diversity and because of the very high cost of such testing. Stratification by an immune response is even more complex because of innumerable peculiarities in functioning of the immune system. Therefore, if the study has a perfect funding source, stratification by infection status can be possible, but stratification by immune response status will be far from ideal.

Unfortunately, to the best of the authors' knowledge, no genome-wide association studies of the connection between polymorphisms of genes encoding the C-type lectin receptor and $\mathrm{RIG}$-I-like receptors and cancer risk or progression have been performed, and this can be explained by the relative newness of the problem or perhaps by another unknown reason.

Summing up, polymorphisms of genes encoding C-type lectin receptors, RIG-I-like receptors, and proteins of their signaling pathways may be promising targets for oncogenomics and possibly could be used in programs of cancer prevention and early cancer diagnostics in the future. Population and further fundamental studies devoted to their association with cancer risk of progression should shed light on this issue.

\section{Disclosure}

The authors report no conflicts of interest in this work.

\section{References}

1. Kawai T, Akira S. Toll-like receptors and their crosstalk with other innate receptors in infection and immunity. Immunity. 2011;34(5):637-650.

2. Elinav E, Strowig T, Henao-Mejia J, Flavell RA. Regulation of the antimicrobial response by NLR proteins. Immunity. 2011;34(5): 665-679.

3. Osorio F, Reis E, Sousa C. Myeloid C-type lectin receptors in pathogen recognition and host defense. Immunity. 2011;34(5):651-664.

4. Loo YM, Gale M Jr. Immune signaling by RIG-I-like receptors. Immunity. 2011;34(5):680-692.

5. Lamba V, Lamba J, Yasuda K, et al. Hepatic CYP2B6 expression: gender and ethnic differences and relationship to CYP2B6 genotype and CAR (constitutive androstane receptor) expression. $J$ Pharmacol Exp Ther. 2003;307(3):906-922.

6. Tierney MJ, Medcalf RL. Plasminogen activator inhibitor type 2 contains mRNA instability elements within exon 4 of the coding region. Sequence homology to coding region instability determinants in other mRNAs. J Biol Chem. 2001;276(17):13675-13684.

7. Thomas KH, Meyn P, Suttorp N. Single nucleotide polymorphism in $5^{\prime}$-flanking region reduces transcription of surfactant protein B gene in H441 cells. Am J Physiol Lung Cell Mol Physiol. 2006;291(3): L386-L390.

8. Zysow BR, Lindahl GE, Wade DP, Knight BL, Lawn RM. C/T polymorphism in the $5^{\prime}$ untranslated region of the apolipoprotein(a) gene introduces an upstream ATG and reduces in vitro translation. Arterioscler Thromb Vasc Biol. 1995;15(1):58-64.

9. Bell JK, Mullen GE, Leifer CA, Mazzoni A, Davies DR, Segal DM. Leucine-rich repeats and pathogen recognition in toll-like receptors. Trends Immunol. 2003;24(10):528-533.

10. Johnson CM, Lyle EA, Omueti KO, et al. Cutting edge: a common polymorphism impairs cell surface trafficking and functional responses of TLR1 but protects against leprosy. J Immunol. 2007;178(12): $7520-7524$.

11. Chang AH, Parsonnet J. Role of bacteria in oncogenesis. Clin Microbiol Rev. 2010;23(4):837-857.

12. de Martel C, Franceschi S. Infections and cancer: established associations and new hypotheses. Crit Rev Oncol Hematol. 2009;70(3):183-194.

13. Hattori T, Konno S, Hizawa N, et al. Genetic variants in the mannose receptor gene (MRC1) are associated with asthma in two independent populations. Immunogenetics. 2009;61(11-12):731-738.

14. Alter A, de Léséleuc L, Van Thuc N, et al. Genetic and functional analysis of common MRC1 exon 7 polymorphisms in leprosy susceptibility. Hum Genet. 2010;127(3):337-348.

15. Hattori T, Konno S, Takahashi A, et al. Genetic variants in mannose receptor gene (MRC1) confer susceptibility to increased risk of sarcoidosis. BMC Med Genet. 2010;11:151.

16. Xu YF, Liu WL, Dong JQ, et al. Sequencing of DC-SIGN promoter indicates an association between promoter variation and risk of nasopharyngeal carcinoma in cantonese. BMC Med Genet. 2010;11:161.

17. Sakuntabhai A, Turbpaiboon C, Casadémont I, et al. A variant in the CD209 promoter is associated with severity of dengue disease. Nat Genet. 2005;37(5):507-513.

18. Wang L, Chen RF, Liu JW, et al. DC-SIGN (CD209) Promoter -336 A/G polymorphism is associated with dengue hemorrhagic fever and correlated to DC-SIGN expression and immune augmentation. PLoS Negl Trop Dis. 2011;5(1):e934.

19. Barreiro LB, Neyrolles O, Babb CL, et al. Promoter variation in the DC-SIGN-encoding gene CD209 is associated with tuberculosis. PLoS Med. 2006;3(2):e20.

20. Vannberg FO, Chapman SJ, Khor CC, et al. CD209 genetic polymorphism and tuberculosis disease. PLoS One. 2008;3(1):e1388. 
21. Selvaraj P, Alagarasu K, Swaminathan S, Harishankar M, Narendran G. CD209 gene polymorphisms in South Indian HIV and HIV-TB patients. Infect Genet Evol. 2009;9(2):256-262.

22. Núñez C, Rueda B, Martínez A, et al. A functional variant in the CD209 promoter is associated with DQ2-negative celiac disease in the Spanish population. World J Gastroenterol. 2006;12(27):4397-4400.

23. Núñez C, Oliver J, Mendoza JL, et al. CD209 in inflammatory bowel disease: a case-control study in the Spanish population. BMC Med Genet. 2007;8:75.

24. Mezger M, Steffens M, Semmler C, et al. Investigation of promoter variations in dendritic cell-specific ICAM3-grabbing non-integrin (DC-SIGN) (CD209) and their relevance for human cytomegalovirus reactivation and disease after allogeneic stem-cell transplantation. Clin Microbiol Infect. 2008;14(3):228-234.

25. Zheng R, Zhou Y, Qin L, et al. Relationship between polymorphism of DC-SIGN (CD209) gene and the susceptibility to pulmonary tuberculosis in an eastern Chinese population. Hum Immunol. 2011; 72(2):183-186.

26. Koizumi Y, Kageyama S, Fujiyama Y, et al. RANTES -28G delays and DC-SIGN - 139C enhances AIDS progression in HIV type 1-infected Japanese hemophiliacs. AIDS Res Hum Retroviruses. 2007; 23(5):713-719.

27. Kashima S, Rodrigues ES, Azevedo R, et al. DC-SIGN (CD209) gene promoter polymorphisms in a Brazilian population and their association with human T-cell lymphotropic virus type 1 infection. J Gen Virol. 2009;90(Pt 4):927-934.

28. Ryan EJ, Dring M, Ryan CM, et al. Variant in CD209 promoter is associated with severity of liver disease in chronic hepatitis $\mathrm{C}$ virus infection. Hum Immunol. 2010;71(8):829-832.

29. Chan KY, Xu MS, Ching JC, et al. CD209 (DC-SIGN) -336 A > G promoter polymorphism and severe acute respiratory syndrome in Hong Kong Chinese. Hum Immunol. 2010;71(7):702-707.

30. Chan KY, Xu MS, Ching JC, et al. Association of a single nucleotide polymorphism in the CD209 (DC-SIGN) promoter with SARS severity. Hong Kong Med J. 2010;16(5 Suppl 4):37-42.

31. Plantinga TS, Fransen J, Takahashi N, et al. Functional consequences of DECTIN-1 early stop codon polymorphism Y238X in rheumatoid arthritis. Arthritis Res Ther. 2010;12(1):R26.

32. Cunha C, Di Ianni M, Bozza S, et al. Dectin-1 Y238X polymorphism associates with susceptibility to invasive aspergillosis in hematopoietic transplantation through impairment of both recipient- and donordependent mechanisms of antifungal immunity. Blood. 2010;116(24): 5394-5402.

33. Chai LY, de Boer MG, van der Velden WJ, et al. The Y238X stop codon polymorphism in the human $\beta$-glucan receptor Dectin- 1 and susceptibility to invasive aspergillosis. J Infect Dis. 2011;203(5):736-743.

34. Plantinga TS, van der Velden WJ, Ferwerda B, et al. Early stop polymorphism in human Dectin-1 is associated with increased candida colonization in hematopoietic stem cell transplant recipients. Clin Infect Dis. 2009;49(5):724-732.

35. Plantinga TS, Hamza OJ, Willment JA, et al. Genetic variation of innate immune genes in HIV-infected african patients with or without oropharyngeal candidiasis. J Acquir Immune Defic Syndr. 2010; 55(1):87-94
36. Pothlichet J, Burtey A, Kubarenko AV, et al. Study of human RIG-I polymorphisms identifies two variants with an opposite impact on the antiviral immune response. PLoS One. 2009;4(10):e7582.

37. Shigemoto T, Kageyama M, Hirai R, Zheng J, Yoneyama M, Fujita T. Identification of loss of function mutations in human genes encoding RIG-I and MDA5: implications for resistance to type I diabetes. J Biol Chem. 2009;284(20):13348-13354.

38. Ovsyannikova IG, Dhiman N, Haralambieva IH, et al. Rubella vaccineinduced cellular immunity: evidence of associations with polymorphisms in the Toll-like, vitamin A and D receptors, and innate immune response genes. Hum Genet. 2010;127(2):207-221.

39. Ovsyannikova IG, Haralambieva IH, Dhiman N, et al. Polymorphisms in the vitamin A receptor and innate immunity genes influence the antibody response to rubella vaccination. J Infect Dis. 2010;201(2):207-213.

40. Hu J, Nistal-Villán E, Voho A, et al. A common polymorphism in the caspase recruitment domain of RIG-I modifies the innate immune response of human dendritic cells. J Immunol. 2010;185(1):424-432.

41. Shu X, Ji J, Li X, Sundquist J, Sundquist K, Hemminki K. Cancer risk among patients hospitalized for Type 1 diabetes mellitus: a populationbased cohort study in Sweden. Diabet Med. 2010;27(7):791-797.

42. Zendehdel K, Nyrén O, Ostenson CG, Adami HO, Ekbom A, Ye W. Cancer incidence in patients with type 1 diabetes mellitus: a population-based cohort study in Sweden. J Natl Cancer Inst. 2003;95(23): 1797-1800.

43. Swerdlow AJ, Laing SP, Qiao Z, et al. Cancer incidence and mortality in patients with insulin-treated diabetes: a UK cohort study. Br J Cancer. 2005;92(11):2070-2075.

44. Hjalgrim H, Frisch M, Ekbom A, Kyvik KO, Melbye M, Green A. Cancer and diabetes - a follow-up study of two population-based cohorts of diabetic patients. J Intern Med. 1997;241(6):471-475.

45. Bahmanyar S, Montgomery SM, Hillert J, Ekbom A, Olsson T. Cancer risk among patients with multiple sclerosis and their parents. Neurology. 2009;72(13):1170-1177.

46. Nielsen NM, Rostgaard K, Rasmussen S, et al. Cancer risk among patients with multiple sclerosis: a population-based register study. Int J Cancer. 2006;118(4):979-984.

47. Sumelahti ML, Pukkala E, Hakama M. Cancer incidence in multiple sclerosis: a 35-year follow-up. Neuroepidemiology. 2004;23(5):224-227.

48. Midgard R, Glattre E, Grønning M, Riise T, Edland A, Nyland H. Multiple sclerosis and cancer in Norway. A retrospective cohort study. Acta Neurol Scand. 1996;93(6):411-415.

49. Fois AF, Wotton CJ, Yeates D, Turner MR, Goldacre MJ. Cancer in patients with motor neuron disease, multiple sclerosis and Parkinson's disease: record linkage studies. J Neurol Neurosurg Psychiatry. 2010;81(2):215-221.

50. Pothlichet J, Niewold TB, Vitour D, Solhonne B, Crow MK, Si-Tahar M. A loss-of-function variant of the antiviral molecule MAVS is associated with a subset of systemic lupus patients. EMBO Mol Med. 2011;3(3): $142-152$.

51. Liu X, Jiao Y, Wen X, et al. Possible association of VISA gene polymorphisms with susceptibility to systemic lupus erythematosus in Chinese population. Mol Biol Rep. 2011;38(7):4583-4588.
Cancer Management and Research

\section{Publish your work in this journal}

Cancer Management and Research is an international, peer-reviewed open access journal focusing on cancer research and the optimal use of preventative and integrated treatment interventions to achieve improved outcomes, enhanced survival and quality of life for the cancer patient The journal welcomes original research, clinical \& epidemiological

\section{Dovepress}

studies, reviews \& evaluations, guidelines, expert opinion \& commentary, case reports \& extended reports. The manuscript management system is completely online and includes a very quick and fair peerreview system, which is all easy to use. Visit http://www.dovepress.com/ testimonials.php to read real quotes from published authors. 